\title{
Lambert-Eaton myasthenic syndrome as a cause of persistent neuromuscular weakness after a mediastinoscopic biopsy -A case report-
}

\author{
Cheol Jin Lee ${ }^{1}$, Se Hun Lim ${ }^{1}$, Chee Mahn Shin ${ }^{1}$, Young Jae Kim, Young Kyun Choe ${ }^{1}$, Soon Ho Cheong ${ }^{1}$, \\ Kun Moo Lee ${ }^{1}$, Jeong Han Lee ${ }^{1}$, Young Hwan Kim ${ }^{1}$, Kwang Rae Cho ${ }^{1}$, Sang Eun Lee ${ }^{1}$, and Jong Suk Bae ${ }^{2}$ \\ Departments of ${ }^{1}$ Anesthesiology and Pain Medicine, ${ }^{2}$ Neurology, Busan Paik Hospital, College of Medicine, Inje University, Busan, Korea
}

There are many causes of prolonged postoperative muscle weakness, including drugs, residual anesthetics, cerebrovascular events, electrolyte imbalance, hypothermia, and neuromuscular disease. Neuromuscular diseases are relatively rare, with the most common being myasthenia gravis and Lambert-Eaton myasthenic syndrome (LEMS). We report an unusual case in which a patient who was given a muscle relaxant during mediastinoscopy developed postoperative muscle weakness that was ultimately diagnosed as secondary to LEMS. (Korean J Anesthesiol 2010; 59: 45-48)

Key Words: Lambert-Eaton myasthenic syndrome, Neuromuscular disease, Postoperative muscular weakness.

In the clinical setting, postoperative muscle weakness is usually attributed to residual anesthetic effects or prolonged neuromuscular blockade. The differential diagnosis of postoperative muscle weakness includes residual anesthetic, cerebrovascular events, electrolyte imbalance, hypothermia, and neuromuscular disease. Musculoskeletal disorders are relatively rare, with the most common being myasthenia gravis and Lambert-Eaton myasthenic syndrome (LEMS) [1,2].

LEMS is a rare neuromuscular junction disease [1]. The clinical features described by Rooke et al. [3] and O'Neill et al. [4] are proximal weakness, especially in the lower limbs, with diminished tendon reflexes and post-tetanic potentiation.
Autonomic symptoms are often reported, including pupil abnormalities, dry eyes and mouth, and erectile dysfunction [5]. Often, the signs and symptoms of LEMS are mistaken for myasthenia gravis and therefore the workup is misdirected [1].

We present a rare case of postoperative prolonged neuromuscular weakness in a previously asymptomatic patient. He was subsequently diagnosed with LEMS.

\section{Case Report}

A 72-year-old man, $169 \mathrm{~cm}$ tall and weighing $56 \mathrm{~kg}$, complained of general weakness and the diagnostic workup

Received: July 8, 2009. Revised: 1st, July 14, 2009; 2nd, August 6, 2009. Accepted: August 7, 2009.

Corresponding author: Se Hun Lim, M.D., Department of Anesthesiology and Pain Medicine, Busan Paik Hospital, College of Medicine, Inje University, 633-615, Gaegeum-dong, Busanjin-gu, Busan 614-735, Korea. Tel: 82-51-890-6964, Fax: 82-51-891-1455, E-mail: anespc@medimail.co.kr This work was supported by the 2008 Inje University Research Grant.

(c) This is an open-access article distributed under the terms of the Creative Commons Attribution Non-Commercial License (http:// creativecommons.org/licenses/by-nc/3.0/), which permits unrestricted non-commercial use, distribution, and reproduction in any medium, provided the original work is properly cited. 
suggested lung cancer with mediastinal lymphadenopathy and syndrome of inappropriate secretion of anti-diuretic hormone (SIADH). To obtain a tissue diagnosis, the patient was scheduled for a mediastinoscopic biopsy.

He had smoked two packs of cigarettes a day for 55 years and had drunk one-and-a-half bottles of soju a day for 50 years. There was no history of neuromuscular disorders and no family history of neuromuscular disease. Four years earlier, he had complained of vertigo and gait disturbance for 1 month and a neurologist had suspected of encephalitis.

Preoperatively, the routine laboratory values were within normal limits, except for hyponatremia ( $125 \mathrm{mEq} / \mathrm{L})$. He had no symptoms or signs of hyponatremia, such as nausea, vomiting, visual disturbances, depressed level of consciousness, agitation, confusion, muscle cramps, weakness, etc. After a nephrology consultation, we decided to perform the scheduled biopsy based on the absence of symptoms and signs associated with hyponatremia and the need to obtain a tissue biopsy to decide on treatment. Pulmonary function tests showed small airway disease.

In the operating room, routine monitoring was used, although neuromuscular transmissions were not monitored. General anesthesia was induced with propofol and succinylcholine. After tracheal intubation, anesthesia was maintained with inhaled sevoflurane $1.5-3$ vol\% and $50 \%$ nitrous oxide in oxygen. Intravenous vecuronium $4 \mathrm{mg}$ was administered for muscle relaxation. Perioperative fluid administration was restricted due to the SIADH. The biopsy was obtained and the pathology showed small cell carcinoma of the lung. It took about 1 hour to incision closure from the time vecuronium was given.

At the end of the procedure, intravenous glycopyrrolate 0.4 mg and pyridostigmine $15 \mathrm{mg}$ were administered to reverse the blockade, but the patient remained motionless and could not breathe spontaneously 40 minutes after reversal. Therefore, we administered atropine $0.5 \mathrm{mg}$ and neostigmine $1.0 \mathrm{mg}$ intravenously in case the dose of the reversal agent had been inadequate. However, there was no improvement in neuromuscular function. His pupils were equal and reactive. The oral body temperature was $36.2^{\circ} \mathrm{C}$ and no antibiotics known to cause neuromuscular blockade had been administered.

We monitored neuromuscular function by attaching a nerve stimulator (TOF-Watch ${ }^{\circledR}$, Organon Ireland Ltd., Dublin, Ireland). There was no twitch response to TOF stimulation, although there was a very weak response to tetanic stimulation at 50 $\mathrm{Hz}$ for 5 seconds with no post-tetanic potentiation. A blood sample was drawn to check the serum electrolytes. There were no abnormal values, other than a serum sodium that was lower than the preoperative level. We thought that sympathetic activation due to the operation might have led to significantly increased levels of $\mathrm{ADH}$ in the absence of volume constriction. We began to correct the hyponatremia with 3\% hypertonic saline, slowly, to prevent cerebral edema or central pontine myelinolysis.

About 2 hours after the end of the procedure, he began to move his fingers slightly and there were four twitch responses to TOF stimulation. However, there was no voluntary respiratory movement or eye opening. We believed that he was alert because he could move his fingers in response to verbal commands.

He was taken to the postanesthesia care unit and placed on a mechanical ventilator. With time, his hyponatremia improved slowly to within the normal range and his neuromuscular function improved. He could move his forearm and hands, but lacked sufficient muscle strength to breathe spontaneously and be extubated. We began to suspect a neuromuscular transmission disorder and a consultation with a neurologist established the diagnosis of LEMS by electromyography (Fig. 1). He was given anticholinesterase via a nasogastric tube, but there was no improvement in neuromuscular function. Two weeks postoperatively, all the laboratory values were within normal limits. He could sit up and write letters. However, weaning from the mechanical ventilator was impossible. He was discharged with a portable ventilator.

\section{Discussion}

We described a patient with LEMS who presented with inadequate recovery from muscular blockade and could not be extubated after a mediastinoscopic biopsy. We suspected hyponatremia as the cause of the prolonged muscular weakness. However, inadequate neuromuscular recovery persisted after correcting the hyponatremia. In this case, we could not find other causes of inadequate recovery from muscular blockade. After a neurology consultation, he was diagnosed as LEMS.

LEMS is an autoimmune presynaptic disorder of neuromuscular transmission [5]. Immunoglobulin G auto-antibodies attack the prejunctional voltage-gated calcium channels and a paucity of prejunctional calcium causes decreased release of acetylcholine quanta into the neuromuscular cleft, resulting in skeletal muscle weakness $[4,6]$. The proximal lower extremity muscles tend to be more susceptible than those in the upper extremity $[4,6,7]$. Skeletal muscle strength often improves with repetitive movement or exercise as more quanta of acetylcholine are released into the neuromuscular junction, but is unresponsive to anticholinesterase medications $[4,7]$. Other symptoms include hypo- or areflexia, autonomic dysfunction (impotence, xerostomia, impaired sweating, etc.), and occasionally bulbar dysfunction $[4,7,8]$. 


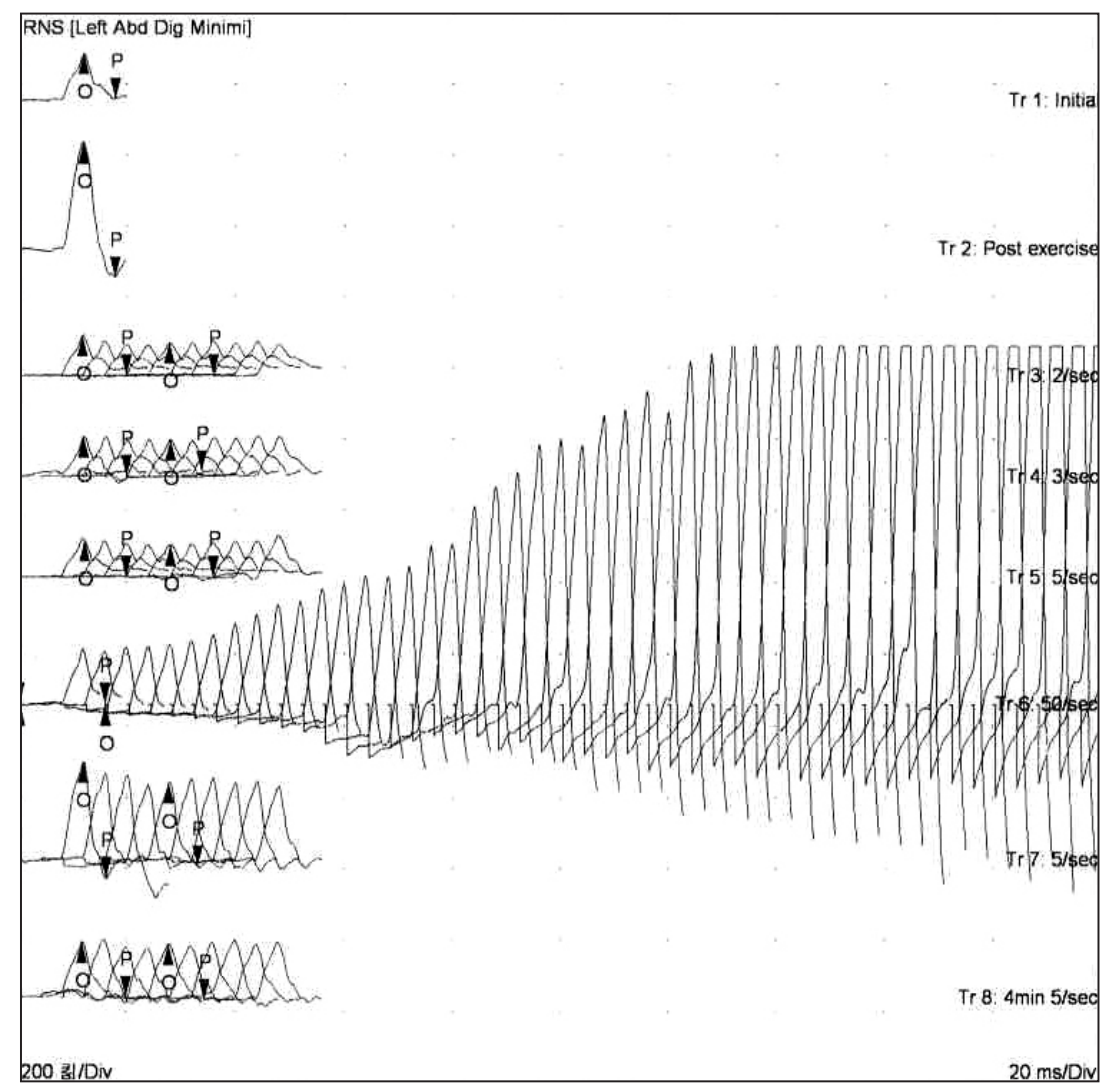

Left Abd Dig Minimi

\begin{tabular}{|c|c|c|c|c|c|c|c|c|c|c|c|}
\hline $\begin{array}{c}\text { Trial } \\
\#\end{array}$ & Label & $\begin{array}{c}\text { Amp } 1 \\
(\mathrm{mV})\end{array}$ & $\begin{array}{c}\text { Amp } 5 \\
(\mathrm{mV})\end{array}$ & $\underset{\text { dif }}{\operatorname{Amp} \%}$ & $\begin{array}{c}\text { Area } 1 \\
\text { (mV-ms) }\end{array}$ & $\begin{array}{c}\text { Area } 5 \\
\text { (mV-ms) }\end{array}$ & $\begin{array}{l}\text { Area } \% \\
\text { dif }\end{array}$ & $\begin{array}{l}\text { Rep } \\
\text { rate }\end{array}$ & $\begin{array}{l}\text { Train } \\
\text { length }\end{array}$ & $\begin{array}{l}\text { Pause time } \\
\text { (min : sec) }\end{array}$ & Comments \\
\hline $\operatorname{Tr} 1$ & Initial & 0.13 & & & 0.50 & & & 1.00 & 1 & $00: 30$ & \\
\hline $\operatorname{Tr} 2$ & Post exercise & 0.38 & & & 1.45 & & & 1.00 & 1 & $01: 00$ & \\
\hline $\operatorname{Tr} 3$ & $2 / \mathrm{sec}$ & 0.11 & 0.09 & -17.6 & 0.00 & 0.00 & 0.0 & 2.00 & 10 & $01: 00$ & \\
\hline $\operatorname{Tr} 4$ & $3 / \mathrm{sec}$ & 0.11 & 0.09 & -19.0 & 0.00 & 0.32 & 0.0 & 3.00 & 10 & $01: 00$ & \\
\hline $\operatorname{Tr} 5$ & $5 / \mathrm{sec}$ & 0.10 & 0.09 & -12.0 & 0.00 & 0.00 & 0.0 & 5.00 & 10 & $01: 00$ & \\
\hline $\operatorname{Tr} 6$ & $50 / \mathrm{sec}$ & 0.00 & 0.00 & 0.0 & 0.00 & 0.00 & 0.0 & 50.00 & 50 & $00: 01$ & \\
\hline $\operatorname{Tr} 7$ & $5 / \mathrm{sec}$ & 0.33 & 0.24 & -27.8 & 0.88 & 0.81 & -7.0 & 5.00 & 10 & $04: 00$ & \\
\hline $\operatorname{Tr} 8$ & $4 \mathrm{~min} 5 / \mathrm{sec}$ & 0.15 & 0.16 & 1.7 & 0.00 & 0.68 & 0.0 & 5.00 & 10 & $00: 00$ & \\
\hline
\end{tabular}

Fig. 1. Repetitive stimulation of the ulnar nerve at low and higher rates. Trial 2 is single post-exercise stimulation that shows a marked incremental response compared with a single stimulation (trial 1). Repetitive stimulation of the ulnar nerve at low rates (Trials 3 to 5 at 2,3 , and $5 \mathrm{~Hz}$, respectively) produced a decremental response in the amplitude of the compound muscle action potentials (CMAPs) recorded from the left abductor digiti minimi muscle. By contrast, stimulation at higher rates (Trial 6; $50 \mathrm{~Hz}$ ) definitely produced an incremental response in the amplitude of the CMAP.

LEMS more commonly affects individuals over 40 years of age and has a male predominance; additionally, the symptoms usually precede tumor diagnosis by over 10 months $[4,7]$.

The diagnosis is confirmed by demonstrating the characteristic electromyographic finding, which is an abnormally low amplitude of the compound muscle action potential evoked by a single nerve stimulus in rested muscle and a progressive increase during high-frequency stimulation or immediately after brief maximal voluntary contraction of the muscle. Our patient had the typical electrophysiologic abnormality (Fig. 1). The diagnosis of LEMS can also be established by the presence of voltage-gated calcium channel antibodies. However, we decided not to check the antibodies because of cost and accessibility problems.

Many reports have associated LEMS with intrathoracic tumors, most notably small cell lung cancer. Our patient also had small cell carcinoma of the lung. Tumors associated with this syndrome include bronchogenic small cell carcinoma 
and breast, ovarian, prostate, and gastrointestinal cancer [4,68]. LEMS should trigger an intensive search for malignancy, especially in patients with a history of tobacco use/abuse [9]. Nevertheless, nearly half of LEMS cases occur in the absence of malignancy $[4,7]$.

The treatment options for LEMS include corticosteroids and immunosuppressive drugs, usually with positive clinical outcomes [10]. Immunosuppressants do have a role in the treatment of LEMS without malignancy [1]. However, when compared to myasthenia gravis, the response to immunosuppression is considerably slower [10]. Since LEMS is mediated by circulating autoantibodies, it is a good candidate for immunomodulation by intravenous immunoglobulins. But, there are no standardized controlled studies [10]. Guanidine hydrochloride inhibits calcium uptake into subcellular organelles, resulting in increased intracellular calcium, which facilitates acetylcholine release from the nerve terminal [7]. However, the side effects of this drug detract from its clinical usefulness. 3,4-diaminopyridine is a drug that selectively blocks potassium in these channels, preventing potassium efflux and prolonging the action potential [7]. This prolongation leaves the voltagegated calcium channel open longer, which results in more calcium entry and more acetylcholine release [7]. In most patients with LEMS, 3,4-diaminopyridine will improve strength [1]. However, 3,4-diaminopyridine was not available at our hospital. Sometimes, LEMS can be combined with myasthenia gravis (overlap myasthenic syndrome) and one patient with this combination responded favorably to treatment with prednisone and anticholinesterase agents [11]. Therefore, we gave our patient anticholinesterase, but there was no improvement in his neuromuscular functions. The possibility of anticholinesterase overload, which could lead to delayed neuromuscular recovery, could be ruled out because there were no symptoms or signs of muscarinic effects of anticholinesterase, such as nausea, vomiting, salivation, diarrhea, and bradycardia [12].

Patients with LEMS have increased sensitivity to both depolarizing and non-depolarizing muscle relaxants; however, the duration of block prolongation is unknown $[3,4,7]$. Therefore, one must be careful with the use of depolarizing or nondepolarizing muscle relaxants perioperatively.

Patients with LEMS have varying degrees of postoperative neuromuscular recovery. One patient was given succinylcholine as part of anesthesia and was extubated postoperatively; two days postoperatively, he developed respiratory failure and required mechanical ventilation [13]. Another patient given suxamethonium and D-tubocurarine could not be extubated for about 7 hours [14]. Yet another patient, who underwent a thyroidectomy under general anesthesia with vecuronium, required mechanical ventilation for about 16 hours postoperatively [2]. And another patient was extubated uneventfully about 3 hours after a cisatracurium bolus [15].

Although LEMS is a rare neuromuscular junction disease, it should be considered as one of the causes of inadequate neuromuscular recovery postoperatively, especially in patients with small cell lung cancer. Monitoring neuromuscular function in the operative period is essential for evaluating the differential diagnosis of inadequate recovery from neuromuscular blockade.

\section{References}

1. Weimer MB, Wong J. Lambert-Eaton myasthenic syndrome. Curr Treat Options Neurol 2009; 11: 77-84.

2. Bui PK, Kuczkowski KM, Moeller-Bertram T, Sanchez RA. New onset Lambert-Eaton myasthenic syndrome as an unexpected cause of delayed recovery from general anesthesia after thyroidectomy. Ann Fr Anesth Reanim 2004; 23: 926-7.

3. Rooke ED, Eaton LM, Lambert EH, Hodgson CH. Myasthenia and malignant intrathoracic tumor. Med Clin North Am 1960; 44: 97788.

4. O'Neill JH, Murray NM, Newsom-Davis J. The Lambert-Eaton myasthenic syndrome. A review of 50 cases. Brain 1988; 111: 57796.

5. Maddison P, Newsom-Davis J. Treatment for Lambert-Eaton myasthenic syndrome. Cochrane Database Syst Rev 2003; 2: CD003279.

6. Greenberg DA. Calcium channels in neurological disease. Ann Neurol 1997; 42: 275-82.

7. Sanders DB. Lambert-Eaton myasthenic syndrome: clinical diagnosis, immune-mediated mechanisms, and update on therapies. Ann Neurol 1995; 37(Suppl 1): S63-73.

8. Blumenfeld AM, Recht LD, Chad DA, DeGirolami U, Griffin T, Jaeckle KA. Coexistence of Lambert-Eaton myasthenic syndrome and subacute cerebellar degeneration: differential effects of treatment. Neurology 1991; 41: 1682-5.

9. Brueck M, Vogel S, Waeger S, Braig G, Kramer W. Lambert-Eaton myasthenic syndrome with acute respiratory failure associated with small cell lung cancer. Dtsch Med Wochenschr 2004; 129: 193-6.

10. Antozzi C. Myasthenia gravis and myasthenic syndrome. Neurol Sci 2003; 24(Suppl 4): S260-3.

11. Roohi F, Smith PR, Bergman M, Baig MA, Sclar G. A diagnostic and management dilemma: combined paraneoplastic myasthenia gravis and Lambert-Eaton myasthenic syndrome presenting as acute respiratory failure. Neurologist 2006; 12: 322-6.

12. Hetherington KA, Losek JD. Myasthenia gravis: myasthenia vs. cholinergic crisis. Pediatr Emerg Care 2005; 21: 546-8.

13. Gracey DR, Southorn PA. Respiratory failure in Lambert-Eaton myasthenic syndrome. Chest 1987; 91: 716-8.

14. Macdonell RA, Rich JM, Cros D, Shahani BT, Ali HH. The LambertEaton myasthenic syndrome: a cause of delayed recovery from general anesthesia. Arch Phys Med Rehabil 1992; 73: 98-100.

15. Leonovicz BM, Gordon EA, Wass CT. Paraneoplastic syndromes associated with lung cancer: a unique case of concomitant subacute cerebellar degeneration and Lambert-Eaton myasthenic syndrome. Anesth Analg 2001; 93: 1557-9. 